OPEN ACCESS

Edited by:

Masashi Maekawa, Ehime University, Japan

Reviewed by:

Yongbin Chen

Kunming Institute of Zoology (CAS),

China

Steven Cheng,

Nanjing Medical University, China

*Correspondence:

Stacey K. Ogden

Stacey.Ogden@stjude.org

Specialty section:

This article was submitted to

Cellular Biochemistry,

a section of the journal

Frontiers in Cell and Developmental

Biology

Received: 15 May 2021

Accepted: 05 July 2021

Published: 30 July 2021

Citation:

Umberger PA and Ogden SK (2021) SPOP and CUL3 Modulate the

Sonic Hedgehog Signal Response

Through Controlled Degradation

of GLI Family Transcription Factors.

Front. Cell Dev. Biol. 9:710295.

doi: 10.3389/fcell.2021.710295

\section{SPOP and CUL3 Modulate the Sonic Hedgehog Signal Response Through Controlled Degradation of GLI Family Transcription Factors}

\author{
Patricia A. Umberger ${ }^{1,2}$ and Stacey K. Ogden ${ }^{1 *}$ \\ ' Department of Cell and Molecular Biology, St. Jude Children's Research Hospital, Memphis, TN, United States, ${ }^{2}$ Integrated \\ Biomedical Sciences Program, University of Tennessee Health Science Center, Memphis, TN, United States
}

The speckle-type POZ protein (SPOP) functions as a guardian of genome integrity and controls transcriptional regulation by functioning as a substrate adaptor for CUL3/RING-type E3 ubiquitin ligase complexes. SPOP-containing CUL3 complexes target a myriad of DNA-binding proteins involved in DNA repair and gene expression, and as such, are essential modulators of cellular homeostasis. GLI transcription factors are effectors of the Hedgehog $(\mathrm{HH})$ pathway, a key driver of tissue morphogenesis and post-developmental homeostasis that is commonly corrupted in cancer. CUL3-SPOP activity regulates amplitude and duration of $\mathrm{HH}$ transcriptional responses by controlling stability of GLI family members. SPOP and GLI co-enrich in phase separated nuclear droplets that are thought to serve as hot spots for CUL3-mediated GLI ubiquitination and degradation. A similar framework exists in Drosophila, in which the Hedgehoginduced MATH (meprin and traf homology) and BTB (bric à brac, tramtrack, broad complex) domain containing protein (HIB) targets the GLI ortholog Cubitus interruptus (Ci) for Cul3-directed proteolysis. Despite this functional conservation, the molecular mechanisms by which $\mathrm{HIB}$ and SPOP contribute to Drosophila and vertebrate $\mathrm{HH}$ signaling differ. In this mini-review we highlight similarities between the two systems and discuss evolutionary divergence in GLI/Ci targeting that informs our understanding of how the GLI transcriptional code is controlled by SPOP and CUL3 in health and disease.

Keywords: Cullin 3, GLI2, GLI3, Hedgehog signaling, developmental signaling, transcription regulation

\section{INTRODUCTION}

Targeted proteolysis maintains cellular homeostasis by providing regulatory control for signal transduction, transcription, metabolism and cell division (Glickman and Ciechanover, 2002; Nandi et al., 2006; Teixeira and Reed, 2013). During embryonic development, the HH pathway functions as a regulator of cell fate determination and signals post-developmentally to maintain tissue homeostasis. HH signaling is controlled in part through proteolysis of downstream transcriptional effectors, Ci in Drosophila and GLI proteins in vertebrates. Degradation is promoted by CullinRING Ligase (CRL) complexes, composed of a cullin scaffold protein, RING subunit, and ubiquitinbound E2 enzyme (Pintard et al., 2004). GLIs are targeted through the ubiquitin-proteasome pathway by both CUL1 and CUL3-CRL complexes. Whereas CRL1 facilitates partial proteolytic 
processing of GLI/Ci in the absence of $\mathrm{HH}$ signal, CRL3 triggers complete GLI/Ci degradation in ligand-stimulated cells (Jiang, 2006; Smelkinson and Kalderon, 2006; Wang et al., 2010). Herein we discuss proteolysis of GLI proteins by the ubiquitinproteasome pathway, focusing on CUL3-mediated degradation via the substrate adaptor SPOP. We examine functional divergence controlling GLI transcription factor degradation between Drosophila and vertebrate systems and comment on important open questions regarding the molecular mechanisms controlling GLI-SPOP association.

\section{UBIQUITIN-PROTEASOME DEGRADATION PATHWAY}

Protein entry into the ubiquitin-proteasome pathway is directed by covalent linkage of ubiquitin to lysine residues of protein targets following stepwise ubiquitin transfer along an E1-E2E3 enzyme cascade (Hochstrasser, 1996). A limited number of E1 ubiquitin activating enzymes service dozens of E2 ubiquitin carrier enzymes and hundreds of E3 ubiquitin ligases (Semple et al., 2003). The largest subclass of E3 ligases are the CRLs. Eight different cullin scaffolding proteins are expressed in human cells (CUL1-3, 4A/B, 5, 7 and 9), each one interacting with specific RING and E2 subunits to form distinct modular ubiquitin ligase complexes (Petroski and Deshaies, 2005). CRL binding to target proteins is directed through recruitment by substate adaptors (Petroski and Deshaies, 2005).

Both CRL1 and CRL3 complexes are crucial modulators of developmental signaling and contribute to $\mathrm{HH}$ pathway regulation. CRL1 complexes target proteins that control cell cycle progression, proliferation, and transcription (Teixeira and Reed, 2013). Their substrate specificity is conferred by associated Skp1 and F-box proteins, which recognize phospho-degrons in substrates such as GLI/Ci (Skaar et al., 2013). CRL3 complexes are more widely used than CRL1 complexes, and degrade proteins involved in myogenesis, neurogenesis, chondrogenesis, osteogenesis, and adipogenesis (Dubiel et al., 2018). CRL3 complexes use BTB superfamily proteins as substrate adaptors (Pintard et al., 2004). The MATH-BTB protein SPOP, which recruits GLIs to CRL3, binds substrates in a multivalent manner to enhance their recruitment into discrete ubiquitination and degradation loci (Zhuang et al., 2009; Marzahn et al., 2016).

\section{SPOP/HIB Is a Multivalent Substrate Adaptor}

SPOP contains three functional domains essential for substrate degradation. These include an amino-terminal MATH domain, a BTB domain and a carboxyl-terminal BACK domain (Figure 1A). The MATH domain binds targets through $\underline{S} P O P$ binding consensus (SBC) motifs (non-polar-polar-Ser-Ser/ThrSer/Thr) (Zhuang et al., 2009). Disordered and elongated SBC motifs within substrates interact with residues of the shallow groove formed by SPOP's MATH domain (Figure 1B; Zhuang et al., 2009). SPOP substrates contain numerous SBCs, which provide multivalent binding capability required for ubiquitination and degradation through CRL3-SPOP.
SPOP's BTB domain facilitates homodimerization and CUL3 binding and its BTB and C-terminal Kelch (BACK) domain drives oligomerization (Figure 1B; Errington et al., 2012). CRL3 complexes containing SPOP oligomerization mutants have diminished ubiquitination capabilities, supporting that multivalent associations are essential for substrate degradation (Zhuang et al., 2009; Marzahn et al., 2016; Bouchard et al., 2018).

Liquid-liquid phase separation (LLPS), or the de-mixing of proteins in solution, drives formation of large protein assemblies called membraneless organelles. These assemblies are thought to compartmentalize distinct biological processes within a cell for efficient interaction between assembly partners (Banani et al., 2017; Shin and Brangwynne, 2017; Boeynaems et al., 2018). A protein's ability to undergo LLPS is driven by multivalent interactions. SPOP can undergo LLPS through homotypic interactions, as exemplified by its enrichment in nuclear speckles. SPOP can also phase separate with binding partners including GLI and DAXX (Kwon et al., 2006; Marzahn et al., 2016). Whereas individual SBCs have weak SPOP binding affinities, the presence of multiple SBC motifs within substrates enhance SPOP association by increasing avidity (Figure 1C; Pierce et al., 2016). This promotes higher-order incorporation of SPOP with substrates into large assemblies that condense to drive LLPS for CRL3-mediated ubiquitination (Errington et al., 2012; Bouchard et al., 2018). Substrates that must phase separate with SPOP for efficient ubiquitination include the GLI proteins. SPOP mutants that prevent higher order oligomerization and LLPS fail to efficiently ubiquitinate GLIs, which triggers $\mathrm{HH}$ gain-of-function phenotypes in vivo (Marzahn et al., 2016).

\section{THE HEDGEHOG SIGNALING PATHWAY}

$\mathrm{HH}$ pathway regulation occurs through a series of inhibitory protein interactions that are reversed by ligand binding (Figure 2). In the absence of $\mathrm{HH}$, its receptor Patched (PTCH) inhibits activity of the signal transducing protein Smoothened (SMO) (Arensdorf et al., 2016). In this off state, GLI2, GLI3 and Ci transcriptional effectors are targeted for partial proteolysis to remove their transcriptional activator domains (Chen et al., 2009; Hui and Angers, 2011). Full-length GLI2/3/Ci (GLI2/3 $\left.{ }^{F L} / \mathrm{Ci}^{F L}\right)$ proteins that are not immediately truncated bind the tumor suppressor SUFU, which prevents transcription activation in the absence of $\mathrm{HH}$ through multiple mechanisms. These include promoting full-length to repressor conversion and preventing nuclear shuttling of $\mathrm{GLI}^{F L} / \mathrm{Ci}^{F L}$ proteins (Barnfield et al., 2005; Chen et al., 2009; Tukachinsky et al., 2010; Shi et al., 2014a). SUFU has also been reported to chaperone $\mathrm{GLI}^{F L} / \mathrm{Ci}^{F L}$ into the nucleus and to recruit histone deacetylase activity to nuclear GLI2/3 through binding the mSin 3 co-repressor complex member SAP18 (Cheng and Bishop, 2002; Paces-Fessy et al., 2004; Sisson et al., 2006; Zhang et al., 2017).

$\mathrm{HH}$ ligand binding to $\mathrm{PTCH}$ activates the pathway by inducing PTCH internalization and degradation, allowing for enrichment of SMO dimers/oligomers on the plasma membrane in Drosophila and in the primary cilium in vertebrates, where it signals for GLI2/3 stabilization and activation (Figure 2; 


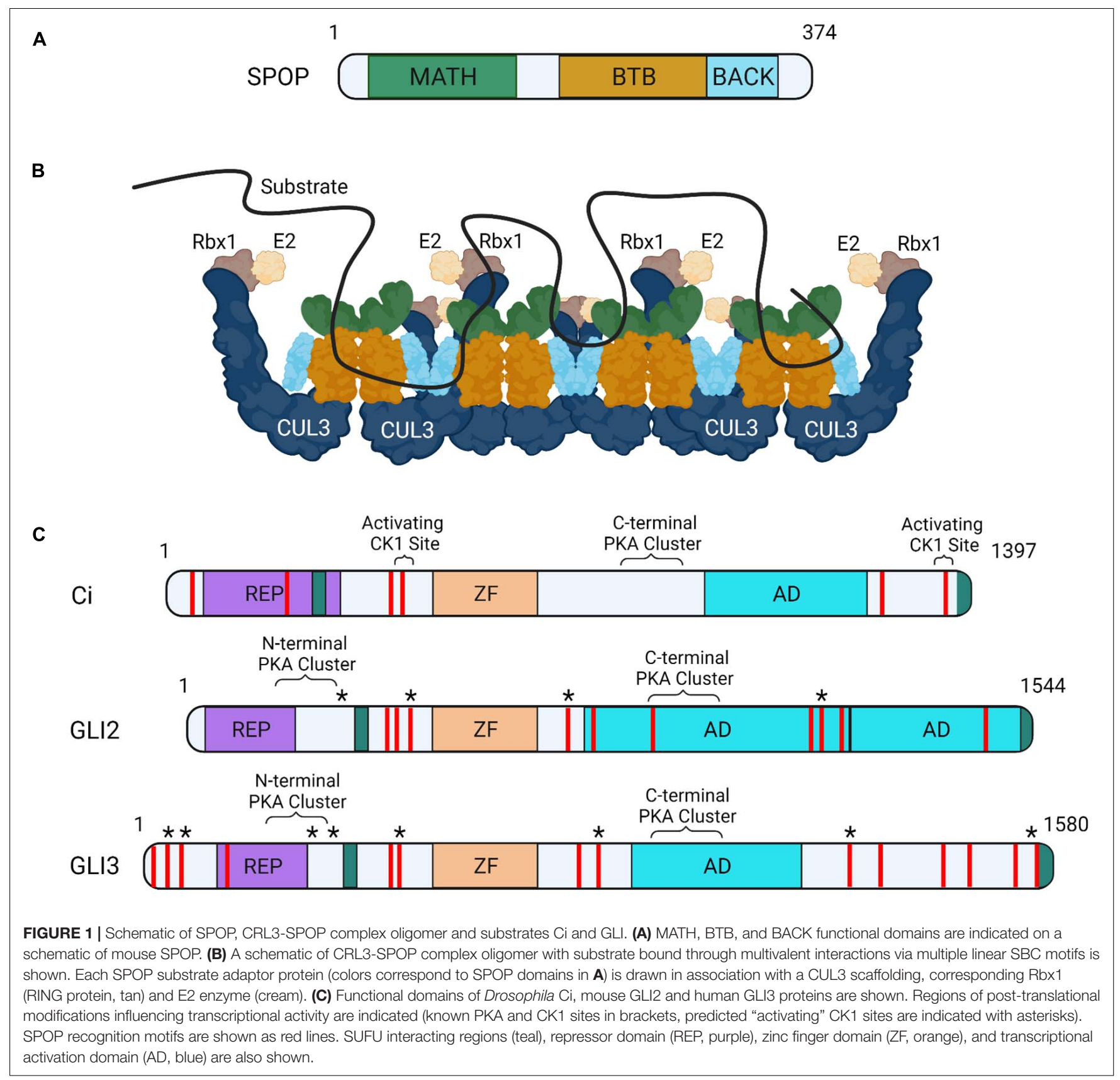

Bangs and Anderson, 2017; Kong et al., 2019). Full-length activated GLI2/3/Ci $\left(\mathrm{GLI} 2 / 3^{A} / \mathrm{Ci}^{A}\right)$ does not efficiently associate with SUFU, and as such, readily enters the nucleus to activate target genes after exiting the primary cilium (Chen et al., 2009; Humke et al., 2010). Whereas Drosophila signaling culminates in activation of a single GLI family member, $\mathrm{Ci}$, vertebrates have three $\mathrm{HH}$ transcriptional effectors, GLI1-3 (Croker et al., 2006; Briscoe and Therond, 2013). As introduced above, stability and activity of GLI2/3 are directly controlled by pathway induction. The gene encoding GLI1 is a transcriptional target of GLI2 $/ 3^{A}$, which is induced in a feed-forward loop to amplify the transcriptional response (Hui and Angers, 2011; Briscoe and
Therond, 2013). Unlike GLI2/3 ${ }^{A}$, GLI1 is not a degradation substrate of SPOP-CUL3 complexes (Zhang et al., 2009), so its regulation will not be further discussed in this review.

\section{Proteolytic Regulation of GLI/Ci}

As introduced above, GLI2/3 and $\mathrm{Ci}$ undergo complex degradation cycles that vary depending upon whether a cell is exposed to ligand. In the absence of ligand, GLI2/3 and Ci are targeted for partial proteolysis by the $26 \mathrm{~S}$ proteasome, converting them into truncated transcriptional repressors (Figure 2, GLI2/3R $/ \mathrm{Ci}^{R}$ ) (Aza-Blanc et al., 1997; Jiang, 2006; Wang and Li, 2006). GLI2/3 and Ci proteins are marked for proteolysis 


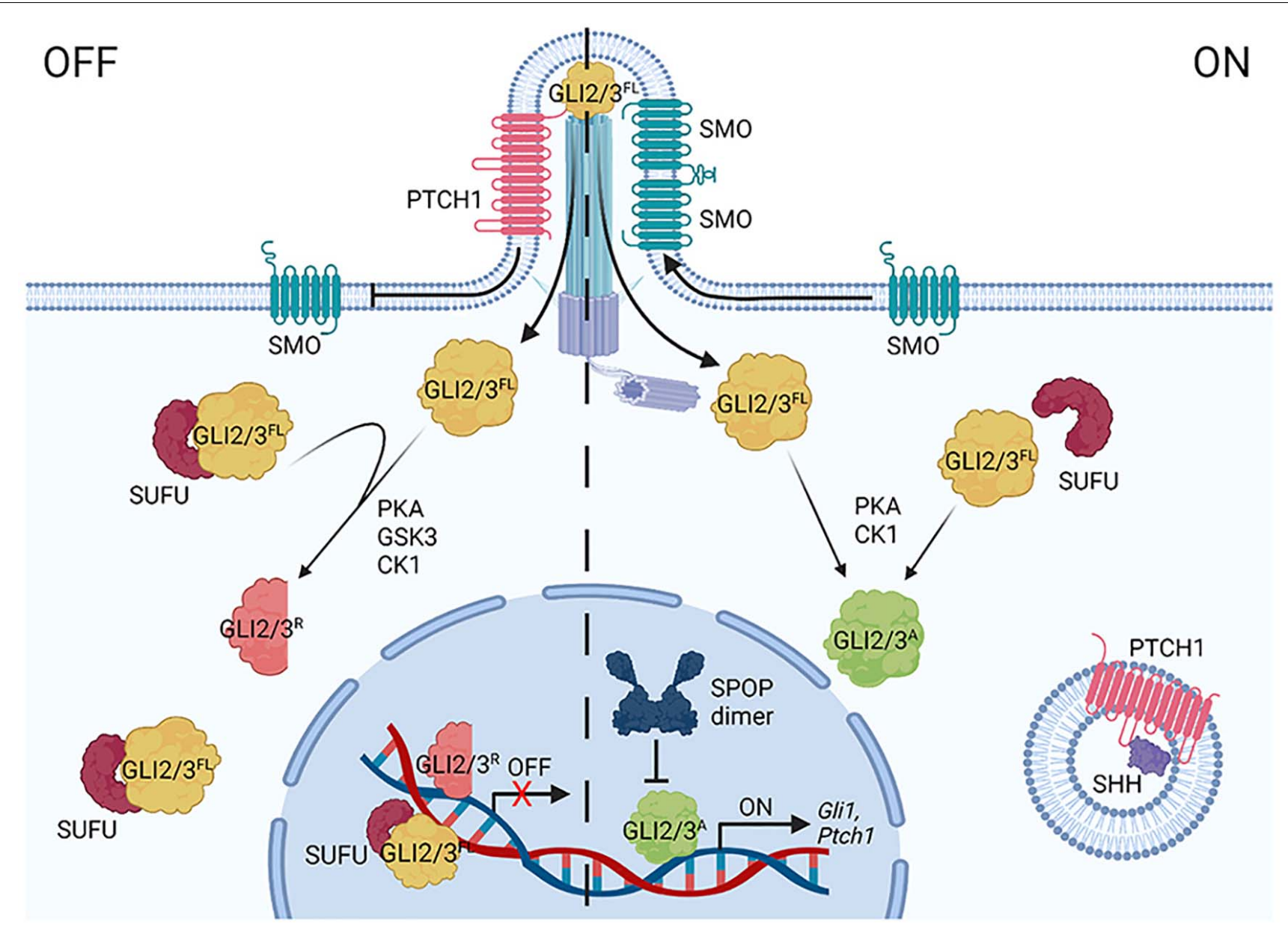

FIGURE 2 | A simplified diagram of the vertebrate HH signaling pathway. In the off state (left), Patched1 (PTCH1) represses Smoothened (SMO) by inhibiting its entry into the primary cilium (PC). GLI2/3 effectors are targeted for partial processing into repressor forms $\left(G L I 2 / 3^{R}\right)$ following sequential phosphorylation via PKA, GSK3( $\beta$ ), and CK1. SUFU binds GLI2/3FL in the cytoplasm and nucleus. SHH binding to PTCH1 (right) triggers its endocytosis, enabling SMO to oligomerize and enrich in the PC. GLI2/3 proteins are activated $\left(G L I 2 / 3^{A}\right.$ ) through alternate phosphorylation events (PKA, CK1), causing dissociation from SUFU and entry into the nucleus to induce target genes (including Gli1 and Ptch1). CRL3-SPOP tunes the GLI response by targeting nuclear GLI2/3 $3^{A}$ for degradation.

through a phosphorylation cascade initiated by cyclic AMPdependent protein kinase (PKA), which leads to subsequent phosphorylation by glycogen synthase kinase 3 [GSK3( $\beta)]$ and casein kinase 1 (CK1) (Figure 1C; Price and Kalderon, 2002; Hui and Angers, 2011). Multi-site phosphorylation promotes GLI2/3 and Ci recruitment to CRL1 by the F-box substrate adapter $\beta$-TrCP/Slimb (Jiang, 2006; Smelkinson and Kalderon, 2006). Pathway activation shifts GLI $2 / 3$ and Ci phosphorylation, attenuating repressor conversion and promoting $\mathrm{GLI} 2 / 3^{A} / \mathrm{Ci}^{A}$ (Figures 1C, 2; Ohlmeyer and Kalderon, 1998; Hui and Angers, 2011; Niewiadomski et al., 2014).

Once in the nucleus, $\mathrm{GLI} 2 / 3^{A} / \mathrm{Ci}^{A}$ are candidates for proteolysis by CRL3-SPOP/HIB complexes that limit the GLI2/3 ${ }^{A} / \mathrm{Ci}^{A}$ transcriptional response (Kent et al., 2006; Zhang et al., 2006). The role for SPOP in regulating stability of GLI family members was first recognized in Drosophila following the discovery that the gene encoding the SPOP homolog HIB is a $\mathrm{Ci}$ transcriptional target. This led to a model in which HIB powered a negative feedback loop to degrade $\mathrm{Ci}$ in cells receiving high-level HH stimulation (Kent et al., 2006; Zhang et al., 2006).

$\mathrm{Ci}$ contains at least six SBCs and GLI2/3 proteins contain upwards of ten (Figure 1C; Zhang et al., 2009). SPOP/HIB can bind SBCs in amino and carboxyl-terminal regions of GLI3 and $\mathrm{Ci}$, and the carboxyl-terminal region of GLI2 (Zhang et al., 2009; Wang et al., 2010). Despite the ability of amino-terminal SBCs to associate with SPOP in the context of the full-length proteins, in vitro over-expression studies of $\mathrm{GLI} 2 / 3^{R}$ mimics suggest $\mathrm{GLI}^{R} / \mathrm{Ci}^{R}$ are not efficiently degraded by CRL3-SPOP (Wang et al., 2010). This differential degradation efficiency of $\mathrm{GLI} 2 / 3^{F L} / \mathrm{Ci}^{F L}$ versus $\mathrm{GLI}^{R} / \mathrm{Ci}^{R}$ may result from affinity differences of amino- and carboxylterminal SBCs. In vitro $\mathrm{Ci}$ binding assays revealed that carboxyl-terminal SBCs recruited HIB more efficiently than amino-terminal SBCs (Zhang et al., 2009). However, amino-terminal SBC-HIB binding efficiency increased with forced $\mathrm{Ci}$ dimerization, suggesting amino-terminal sites 
may cooperate in trans. SBCs in the carboxyl domain of $\mathrm{Ci}$ functioned cooperatively in cis, allowing for high affinity combinatorial binding to HIB independent of Ci dimerization (Zhang et al., 2009).

Another mechanism by which SPOP-GLI2/3 and HIB$\mathrm{Ci}$ binding may be affected is through phosphorylation of serine/threonine residues within SBC motifs. Some GLI2/3 and $\mathrm{Ci}$ SBCs are phosphorylated by the serine/threonine kinase CK1 following pathway activation (Shi et al., 2014b). Although activating CK1 sites in GLI2/3 have not been mapped, phosphorylation by $\mathrm{CK} 1$ is reported to attenuate SPOP/HIB binding to slow $\mathrm{GLI} 2 / 3^{A} / \mathrm{Ci}^{A}$ degradation. Notably, whereas all Ci SBCs are capable of being phosphorylated by CK1, select SBC clusters are phosphorylated more efficiently (Figure 1C). This suggests graded CK1 phosphorylation may provide a mechanism to tune SPOP/HIB binding proportional to $\mathrm{HH}$ exposure (Shi et al., 2014b). SBC phosphorylation in SPOP substrates MacroH2A, Puckered, and Pdx1 prevents SPOP binding, suggesting phosphorylation provides a conserved regulatory mechanism for CRL3-SPOP engagement (Zhuang et al., 2009; Ostertag et al., 2019).

PKA is another serine/threonine kinase with dual roles in GLI2/3 control. In response to SHH, GLI2/3 proteins are phosphorylated by PKA at a series of amino-terminal sites that promote GLI2/3 ${ }^{A}$ (Figure 1C; Niewiadomski et al., 2014). Like GLI, Ci has multiple PKA clusters in its aminoterminus, suggesting ligand-stimulated phosphorylation plays a conserved role in $\mathrm{Ci}^{A}$ generation (Niewiadomski et al., 2014). The activating PKA sites in GLI/Ci are located adjacent to amino-terminal SBCs, but whether they directly impact SPOP/HIB binding is not yet clear. Given the ability of CK1 phosphorylation to directly alter affinity of SBCs for SPOP, it is possible that PKA phosphorylation could have similar effects (Shi et al., 2014b). Alternatively, PKA phosphorylation may alter SPOP binding indirectly by inducing conformational shifts in GLI/Ci that hinder multivalent SPOP/HIB association.

SUFU binds GLI $2 / 3^{F L} / \mathrm{Ci}^{F L}$ proteins in both flies and vertebrates. In addition to buffering $\mathrm{GLI} 2 / 3 / \mathrm{Ci}$ transcriptional activity through this binding, SUFU also buffers against SPOP/HIB. This is because SBCs in GLI2/3/Ci are located adjacent to SUFU recognition sites, which results in SUFU and SPOP/HIB binding GLI2/3/Ci in competitive manners (Dunaeva et al., 2003; Zhang et al., 2009; Seong and Ishii, 2013; Han et al., 2015). A role for SUFU in blunting SPOPmediated GLI2/3 destabilization was delineated in mouse embryo fibroblasts (MEFs) derived from Sufu knockout animals. Reduced levels of both GLI2/3 ${ }^{A}$ and $G L I 3^{R}$ observed in the absence of SUFU were rescued by its re-expression (Chen et al., 2009; Wang et al., 2010). So, while both SUFU and SPOP negatively regulate GLI2/3 activity, they do so through distinct mechanisms. Whereas SPOP limits the duration of GLI $2 / 3^{A}$ transcriptional activity by degrading GLI $2 / 3^{A}$ in the nucleus, SUFU controls the amplitude of the GLI transcriptional response through physical association with GLI to block both CRL3-SPOP degradation and transcriptional activity.

\section{CRL3-HIB REGULATION OF Drosophila HH SIGNALING}

A role for CRL3-HIB in controlling Ci stability was identified through misexpression studies during Drosophila eye and wing development. Ectopic expression of $\mathrm{HIB}$ reduced $\mathrm{Ci}^{F L}$ protein in both tissues, and HIB elimination resulted in $\mathrm{Ci}^{F L}$ accumulation and increased target gene induction (Kent et al., 2006; Zhang et al., 2006). These results led to the model whereby $\mathrm{HIB}$ induction limits $\mathrm{Ci}^{A}$ accumulation and activity in cells receiving high-level $\mathrm{HH}$ stimulation. HIBmediated $\mathrm{Ci}$ degradation in the wing was initially proposed to be spatially restricted due to high-level HIB expression occurring only in cells directly adjacent to a $\mathrm{HH}$ source (Kent et al., 2006; Zhang et al., 2006). However, overexpression studies revealed HIB limits wing overgrowth caused by excessive $\mathrm{Ci}$ transcriptional activity throughout the wing, suggesting its ability to control $\mathrm{Ci}$ is not spatially limited (Zhang et al., 2006).

Because HIB is the only MATH-BTB protein in Drosophila, and it is induced by high-level $\mathrm{HH}$ signaling, it was initially proposed to be a specific modulator of Ci. However, more recent studies demonstrate HIB influences stability of proteins not linked to the $\mathrm{HH}$ cascade including the phosphatase Puckered, and the chromosomal proteins CAL1 and Histone H3 variant CENP-A (Liu et al., 2009; Zhang et al., 2009; Bade et al., 2014). Additionally, a recent report revealed CRL3HIB regulation of $\mathrm{Ci}$ is not as straightforward as indicated by early over-expression studies. CRISPR-generated Drosophila harboring mutations in the endogenous $c i$ allele that disrupt HIB binding develop normally, suggesting HIB is not essential for regulation of $\mathrm{Ci}$ expressed at physiological levels (Little et al., 2020). The cause of these disparate results is not yet clear but may indicate that use of over-expression systems can mask cooperating or redundant mechanisms that control $\mathrm{Ci}$ activity. This raises the possibility that HIB may not function as a specific feed-back regulator of $\mathrm{HH}$ signaling in flies but may instead prevent excessive signaling when $\mathrm{Ci}$ accumulates to uncharacteristically high levels.

\section{CRL3-SPOP REGULATION OF VERTEBRATE HH SIGNALING}

Despite the conserved role of CRL3-SPOP/HIB in controlling the amplitude and duration of $\mathrm{HH}$ responses through ubiquitination and degradation of GLI $2 / 3^{A} / \mathrm{Ci}^{A}$, divergence between the systems has occurred. Most notably, whereas CRL3-HIB degrades $\mathrm{Ci}^{A}$ independent of tissue context, CRL3-SPOP regulation of GLI2/3 ${ }^{A}$ occurs in tissue and context-dependent manners (Cai and Liu, 2016, 2017; Coquenlorge et al., 2019; Yin et al., 2019). In the neural tube (NT), Sonic Hedgehog (SHH) instructs organization of ventral neuronal progenitor domains through balancing activity of $\mathrm{GLI}^{A}$ and $\mathrm{GLI}^{R}$ proteins (Persson et al., 2002; Lei et al., 2004). Despite the ability of CRL3-SPOP to directly target GLI $2 / 3^{A}$, the ventral NT is appropriately patterned in Spop knockout mice, suggesting SPOP is not 
an essential regulator of GLI2/3 in this tissue (Cai and Liu, 2017). However, individually targeting Gli2 or Gli3 in Spop ${ }^{-1-}$ mice revealed SPOP specifically impacts $\mathrm{GLI}^{A}$ during NT development. Failure of ventral NT fate induction observed in $\mathrm{Gli2}^{-/-}$animals was rescued in Gli2/Spop double knockout animals, indicating SPOP loss may increase compensatory GLI3 ${ }^{A}$ activity in the absence of GLI2. Indeed, increases in both GLI3 ${ }^{F L}$ and $\mathrm{GLI}^{R}$ were observed in $S p o p^{-/-}$tissue (Cai and Liu, 2017). Importantly, comparison of $\mathrm{Gli3}^{-/-}$and Gli3/Spop double knockout embryos revealed similar NT defects. This supports that GLI3 is likely the main target of SPOP in the NT, and that CRL3-SPOP is not a key modulator of GLI2 ${ }^{A}$ protein levels in this tissue (Cai and Liu, 2017).

Spop ${ }^{-/-}$mice reveal that, in bone, SPOP plays a positive regulatory role that promotes GLI3 activity downstream of Indian Hedgehog ( $\mathrm{IHH})$. The positive contribution of SPOP was identified through skeletal target gene analysis, which revealed reduced $\mathrm{IHH}$ target gene induction and decreased osteoblast and chondrocyte differentiation in Spop ${ }^{-/-}$metatarsal and limb tissues (Cai and Liu, 2016). Similar to what was observed in the Spop ${ }^{-/-}$NT, effects on GLI2 were modest in the skeleton. Intriguingly, SPOP loss impacted $\mathrm{IHH}$ signaling by increasing $\mathrm{GLI}^{R}$, rather than by influencing $\mathrm{GLI}^{A}{ }^{A}$ (Cai and Liu, 2016, 2017). Whether this indicates direct targeting of GLI $3^{R}$ by SPOP in skeletal tissue, or if $\mathrm{GLI} 3^{R}$ accumulation was the byproduct of increased full-length GLI3, is not yet clear. CRL3-SPOP may directly target $\mathrm{GLI}^{R}$ because amino-terminal constructs that mimic $\mathrm{GLI} 3^{R} / \mathrm{Ci}^{R}$ can be ubiquitinated by CRL3-SPOP/HIB in in vitro assays (Zhang et al., 2006; Cai and Liu, 2016; Marzahn et al., 2016). Further investigation is needed to clarify whether $\mathrm{GLI} 3^{R} / \mathrm{Ci}^{R}$ are bona fide physiological targets of CRL3SPOP.

Specific effects on GLI2 ${ }^{A}$ by CRL3-SPOP were identified in the mesenchymal niche and brain following combined targeting of Sufu with Spop. Gut mesenchymal development depends upon coordinated activity of $\mathrm{SHH}, \mathrm{IHH}$ and plateletderived growth factor signaling between mesenchymal and epithelial layers (Mao et al., 2010; Walton et al., 2012). Activation of downstream signaling by conditional deletion of Sufu in gut mesenchyme resulted in enlarged stomachs, shortened intestines, significant mesenchymal tissue expansion, and perinatal lethality. Whereas conditional deletion of Spop in the gut mesenchyme did not yield a developmental phenotype, combining Spop with Sufu deletion exacerbated the Sufu knockout phenotype (Coquenlorge et al., 2019; Yung et al., 2019). In Spop/Sufu'-/- animals, elevated GLI2 led to mesenchymal and epithelial tissue overgrowth and intestinal tumorigenesis, which was corrected by reducing Gli2 gene dosage (Coquenlorge et al., 2019).

In the brain, compound deletion of Spop and Sufu in the cerebellum led to rapid development of highly aggressive medulloblastoma (Yin et al., 2019). Tumors were significantly affected by gene dosage because reduced tumor burden was observed in Gli2 heterozygous Spop;Sufu double knockout animals. Tumors were further reduced by increasing Spop gene dosage. Sufu-/-;Spop+/-;Gli2+/- animals showed normal cerebellar patterning and a significant reduction in tumor incidence (Yin et al., 2019). Combined with the work discussed above, these genetic studies suggest $\mathrm{SHH}$ pathway activity can be controlled through coordinated targeting of GLI2 by CRL3-SPOP and SUFU to scale transcriptional responses downstream of ligand.

\section{CONCLUDING REMARKS}

Complex regulatory events controlling proteolysis of GLI family transcription factors are crucial determinants of $\mathrm{HH}$ target gene regulation in the absence and presence of ligand. Experimental evidence indicates CRL3-SPOP complexes are important players in this process but understanding of the molecular mechanisms and context-dependent cues controlling GLI2/3 recruitment to CRL3 by SPOP is in its infancy. As such, continued genetic, biochemical, and biophysical interrogation of CRL3-SPOP regulation of $\mathrm{HH}$ pathway activity is needed. A focus area that will yield important information about regulation of GLI family proteins will be dissection of signals driving their LLPS with SPOP. Such studies will reveal how LLPS promotes CRL3-mediated elimination of $\mathrm{GLI} 2 / 3^{A}$ and may provide novel therapeutic opportunities for targeting aberrant GLI2/3 transcriptional activity in disease. In addition, continued dissection of the coordination between SUFU and SPOP association with $\mathrm{GLI} 2 / 3 / \mathrm{Ci}$ will be essential to clarify the complex regulatory mechanisms controlling GLI2/3/Ci transcriptional output. Such studies may reveal developmental, tissue, and/or temporal-specific cues that direct differential CRL3-SPOP targeting of GLI that may be amenable to therapeutic intervention.

\section{AUTHOR CONTRIBUTIONS}

PU wrote the manuscript. SO edited the manuscript. Both authors contributed to the article and approved the submitted version.

\section{FUNDING}

This work was supported by R35GM122546 (SO) and by ALSAC of St. Jude Children's Research Hospital. The content was solely the responsibility of the authors and may not represent the official views of the National Institutes of Health.

\section{ACKNOWLEDGMENTS}

We thank members of the Ogden Lab for comments on the manuscript. The figures were created with BioRender.com. We apologize to those whose work was not discussed due to space limitations. 


\section{REFERENCES}

Arensdorf, A. M., Marada, S., and Ogden, S. K. (2016). Smoothened regulation: a tale of two signals. Trends Pharmacol. Sci. 37, 62-72. doi: 10.1016/j.tips.2015.09. 001

Aza-Blanc, P., Ramirez-Weber, F. A., Laget, M. P., Schwartz, C., and Kornberg, T. B. (1997). Proteolysis that is inhibited by hedgehog targets Cubitus interruptus protein to the nucleus and converts it to a repressor. Cell 89, 1043-1053. doi: 10.1016/s0092-8674(00)80292-5

Bade, D., Pauleau, A. L., Wendler, A., and Erhardt, S. (2014). The E3 ligase CUL3/RDX controls centromere maintenance by ubiquitylating and stabilizing CENP-A in a CAL1-dependent manner. Dev. Cell 28, 508-519. doi: 10.1016/j. devcel.2014.01.031

Banani, S. F., Lee, H. O., Hyman, A. A., and Rosen, M. K. (2017). Biomolecular condensates: organizers of cellular biochemistry. Nat. Rev. Mol. Cell Biol. 18, 285-298. doi: 10.1038/nrm.2017.7

Bangs, F., and Anderson, K. V. (2017). Primary cilia and mammalian hedgehog signaling. Cold Spring Harb. Perspect. Biol. 9:a028175. doi: 10.1101/cshperspect. a028175

Barnfield, P. C., Zhang, X., Thanabalasingham, V., Yoshida, M., and Hui, C. C. (2005). Negative regulation of Gli1 and Gli2 activator function by Suppressor of fused through multiple mechanisms. Differentiation 73, 397-405. doi: 10.1111/ j.1432-0436.2005.00042.x

Boeynaems, S., Alberti, S., Fawzi, N. L., Mittag, T., Polymenidou, M., Rousseau, F., et al. (2018). Protein phase separation: a new phase in cell biology. Trends Cell Biol. 28, 420-435. doi: 10.1016/j.tcb.2018.02.004

Bouchard, J. J., Otero, J. H., Scott, D. C., Szulc, E., Martin, E. W., Sabri, N., et al. (2018). Cancer mutations of the tumor suppressor SPOP disrupt the formation of active, phase-separated compartments. Mol. Cell 72, 19.e8-36.e8. doi: 10.1016/j.molcel.2018.08.027

Briscoe, J., and Therond, P. P. (2013). The mechanisms of Hedgehog signalling and its roles in development and disease. Nat. Rev. Mol. Cell Biol. 14, 416-429. doi: $10.1038 / \mathrm{nrm} 3598$

Cai, H., and Liu, A. (2016). Spop promotes skeletal development and homeostasis by positively regulating Ihh signaling. Proc. Natl. Acad. Sci. U.S.A. 113, 1475114756. doi: 10.1073/pnas.1612520114

Cai, H., and Liu, A. (2017). Spop regulates Gli3 activity and Shh signaling in dorsoventral patterning of the mouse spinal cord. Dev. Biol. 432, 72-85. doi: 10.1016/j.ydbio.2017.04.002

Chen, M. H., Wilson, C. W., Li, Y. J., Law, K. K., Lu, C. S., Gacayan, R., et al. (2009). Cilium-independent regulation of Gli protein function by Sufu in Hedgehog signaling is evolutionarily conserved. Genes Dev. 23, 1910-1928. doi: 10.1101/ gad.1794109

Cheng, S. Y., and Bishop, J. M. (2002). Suppressor of Fused represses Gli-mediated transcription by recruiting the SAP18-mSin3 corepressor complex. Proc. Natl. Acad. Sci. U.S.A. 99, 5442-5447. doi: 10.1073/pnas.082096999

Coquenlorge, S., Yin, W. C., Yung, T., Pan, J., Zhang, X., Mo, R., et al. (2019). GLI2 Modulated by SUFU and SPOP induces intestinal stem cell niche signals in development and tumorigenesis. Cell Rep. 27, 3006.e4-3018.e4. doi: 10.1016/ j.celrep.2019.05.016

Croker, J. A., Ziegenhorn, S. L., and Holmgren, R. A. (2006). Regulation of the Drosophila transcription factor, Cubitus interruptus, by two conserved domains. Dev. Biol. 291, 368-381. doi: 10 . 1016/j.ydbio.2005.12.020

Dubiel, W., Dubiel, D., Wolf, D. A., and Naumann, M. (2018). Cullin 3-Based ubiquitin ligases as master regulators of mammalian cell differentiation. Trends Biochem. Sci. 43, 95-107. doi: 10.1016/j.tibs.2017.11.010

Dunaeva, M., Michelson, P., Kogerman, P., and Toftgard, R. (2003). Characterization of the physical interaction of Gli proteins with SUFU proteins. J. Biol. Chem. 278, 5116-5122. doi: 10. 1074/jbc.M209492200

Errington, W. J., Khan, M. Q., Bueler, S. A., Rubinstein, J. L., Chakrabartty, A., and Prive, G. G. (2012). Adaptor protein self-assembly drives the control of a cullin-RING ubiquitin ligase. Structure 20, 1141-1153. doi: 10.1016/j.str.2012. 04.009

Glickman, M. H., and Ciechanover, A. (2002). The ubiquitin-proteasome proteolytic pathway: destruction for the sake of construction. Physiol. Rev. 82, 373-428. doi: 10.1152/physrev.00027.2001
Han, Y., Shi, Q., and Jiang, J. (2015). Multisite interaction with Sufu regulates $\mathrm{Ci} / \mathrm{Gli}$ activity through distinct mechanisms in Hh signal transduction. Proc. Natl. Acad. Sci. U.S.A. 112, 6383-6388. doi: 10.1073/pnas. 1421628112

Hochstrasser, M. (1996). Ubiquitin-dependent protein degradation. Annu. Rev. Genet. 30, 405-439. doi: 10.1146/annurev.genet.30.1.405

Hui, C. C., and Angers, S. (2011). Gli proteins in development and disease. Annu. Rev. Cell Dev. Biol. 27, 513-537. doi: 10.1146/annurev-cellbio-092910-154048

Humke, E. W., Dorn, K. V., Milenkovic, L., Scott, M. P., and Rohatgi, R. (2010). The output of Hedgehog signaling is controlled by the dynamic association between Suppressor of Fused and the Gli proteins. Genes Dev. 24, 670-682. doi: 10.1101/gad.1902910

Jiang, J. (2006). Regulation of Hh/Gli signaling by dual ubiquitin pathways. Cell Cycle 5, 2457-2463. doi: 10.4161/cc.5.21.3406

Kent, D., Bush, E. W., and Hooper, J. E. (2006). Roadkill attenuates Hedgehog responses through degradation of Cubitus interruptus. Development 133, 20012010. doi: $10.1242 /$ dev.02370

Kong, J. H., Siebold, C., and Rohatgi, R. (2019). Biochemical mechanisms of vertebrate hedgehog signaling. Development 146:dev166892. doi: 10.1242/dev. 166892

Kwon, J. E., La, M., Oh, K. H., Oh, Y. M., Kim, G. R., Seol, J. H., et al. (2006). BTB domain-containing speckle-type POZ protein (SPOP) serves as an adaptor of Daxx for ubiquitination by Cul3-based ubiquitin ligase. J. Biol. Chem. 281, 12664-12672. doi: 10.1074/jbc.M600204200

Lei, Q., Zelman, A. K., Kuang, E., Li, S., and Matise, M. P. (2004). Transduction of graded Hedgehog signaling by a combination of Gli2 and Gli3 activator functions in the developing spinal cord. Development 131, 3593-3604. doi: 10.1242/dev.01230

Little, J. C., Garcia-Garcia, E., Sul, A., and Kalderon, D. (2020). Drosophila hedgehog can act as a morphogen in the absence of regulated $\mathrm{Ci}$ processing. Elife 9:e61083. doi: 10.7554/eLife.61083

Liu, J., Ghanim, M., Xue, L., Brown, C. D., Iossifov, I., Angeletti, C., et al. (2009). Analysis of Drosophila segmentation network identifies a JNK pathway factor overexpressed in kidney cancer. Science 323, 1218-1222. doi: 10.1126/science. 1157669

Mao, J., Kim, B. M., Rajurkar, M., Shivdasani, R. A., and McMahon, A. P. (2010). Hedgehog signaling controls mesenchymal growth in the developing mammalian digestive tract. Development 137, 1721-1729. doi: 10.1242/dev. 044586

Marzahn, M. R., Marada, S., Lee, J., Nourse, A., Kenrick, S., Zhao, H., et al. (2016). Higher-order oligomerization promotes localization of SPOP to liquid nuclear speckles. EMBO J. 35, 1254-1275. doi: 10.15252/embj.201593169

Nandi, D., Tahiliani, P., Kumar, A., and Chandu, D. (2006). The ubiquitinproteasome system. J. Biosci. 31, 137-155. doi: 10.1007/BF02705243

Niewiadomski, P., Kong, J. H., Ahrends, R., Ma, Y., Humke, E. W., Khan, S., et al. (2014). Gli protein activity is controlled by multisite phosphorylation in vertebrate Hedgehog signaling. Cell Rep. 6, 168-181. doi: 10.1016/j.celrep.2013. 12.003

Ohlmeyer, J. T., and Kalderon, D. (1998). Hedgehog stimulates maturation of Cubitus interruptus into a labile transcriptional activator. Nature 396, 749-753. doi: $10.1038 / 25533$

Ostertag, M. S., Messias, A. C., Sattler, M., and Popowicz, G. M. (2019). The structure of the SPOP-Pdx1 interface reveals insights into the phosphorylationdependent binding regulation. Structure 27, 327.e3-334.e3. doi: 10.1016/j.str. 2018.10.005

Paces-Fessy, M., Boucher, D., Petit, E., Paute-Briand, S., and Blanchet-Tournier, M. F. (2004). The negative regulator of Gli, Suppressor of fused (Sufu), interacts with SAP18, Galectin3 and other nuclear proteins. Biochem J 378(Pt 2), 353362. doi: 10.1042/BJ20030786

Persson, M., Stamataki, D., te Welscher, P., Andersson, E., Bose, J., Ruther, U., et al. (2002). Dorsal-ventral patterning of the spinal cord requires Gli3 transcriptional repressor activity. Genes Dev. 16, 2865-2878. doi: 10.1101/gad. 243402

Petroski, M. D., and Deshaies, R. J. (2005). Function and regulation of cullin-RING ubiquitin ligases. Nat, Rev. Mol. Cell Biol. 6, 9-20. doi: 10.1038/nrm1547

Pierce, W. K., Grace, C. R., Lee, J., Nourse, A., Marzahn, M. R., Watson, E. R., et al. (2016). Multiple weak linear motifs enhance recruitment and processivity in SPOP-mediated substrate ubiquitination. J. Mol. Biol. 428, 1256-1271. doi: 10.1016/j.jmb.2015.10.002 
Pintard, L., Willems, A., and Peter, M. (2004). Cullin-based ubiquitin ligases: Cul3BTB complexes join the family. EMBO J. 23, 1681-1687. doi: 10.1038/sj.emboj. 7600186

Price, M. A., and Kalderon, D. (2002). Proteolysis of the Hedgehog signaling effector Cubitus interruptus requires phosphorylation by Glycogen Synthase Kinase 3 and Casein Kinase 1. Cell 108, 823-835. doi: 10.1016/s0092-8674(02) 00664-5

Semple, C. A., Group, R. G., and Members, G. S. L. (2003). The comparative proteomics of ubiquitination in mouse. Genome Res. 13, 1389-1394. doi: 10. 1101/gr.980303

Seong, K. H., and Ishii, S. (2013). Su(fu) switches Rdx functions to fine-tune hedgehog signaling in the Drosophila wing disk. Genes Cells 18, 66-78. doi: 10.1111/gtc. 12018

Shi, Q., Han, Y., and Jiang, J. (2014a). Suppressor of fused impedes Ci/Gli nuclear import by opposing Trn/Kapbeta2 in Hedgehog signaling. J. Cell Sci. 127(Pt 5), 1092-1103. doi: 10.1242/jcs. 142828

Shi, Q., Li, S., Li, S., Jiang, A., Chen, Y., and Jiang, J. (2014b). Hedgehog-induced phosphorylation by CK1 sustains the activity of Ci/Gli activator. Proc. Natl. Acad. Sci. U.S.A. 111, E5651-E5660. doi: 10.1073/pnas.1416652111

Shin, Y., and Brangwynne, C. P. (2017). Liquid phase condensation in cell physiology and disease. Science 357:eaaf4382. doi: 10.1126/science.aaf4382

Sisson, B. E., Ziegenhorn, S. L., and Holmgren, R. A. (2006). Regulation of Ci and $\mathrm{Su}(\mathrm{fu})$ nuclear import in Drosophila. Dev. Biol. 294, 258-270. doi: 10.1016/j. ydbio.2006.02.050

Skaar, J. R., Pagan, J. K., and Pagano, M. (2013). Mechanisms and function of substrate recruitment by F-box proteins. Nat. Rev. Mol. Cell Biol. 14, 369-381. doi: $10.1038 / \mathrm{nrm} 3582$

Smelkinson, M. G., and Kalderon, D. (2006). Processing of the Drosophila hedgehog signaling effector $\mathrm{Ci}-155$ to the repressor $\mathrm{Ci}-75$ is mediated by direct binding to the SCF component Slimb. Curr. Biol. 16, 110-116. doi: 10.1016/j. cub.2005.12.012

Teixeira, L. K., and Reed, S. I. (2013). Ubiquitin ligases and cell cycle control. Annu. Rev. Biochem. 82, 387-414. doi: 10.1146/annurev-biochem-060410-105307

Tukachinsky, H., Lopez, L. V., and Salic, A. (2010). A mechanism for vertebrate Hedgehog signaling: recruitment to cilia and dissociation of SuFu-Gli protein complexes. J. Cell Biol. 191, 415-428. doi: 10. 1083/jcb.201004108

Walton, K. D., Kolterud, A., Czerwinski, M. J., Bell, M. J., Prakash, A., Kushwaha, J., et al. (2012). Hedgehog-responsive mesenchymal clusters direct patterning and emergence of intestinal villi. Proc. Natl. Acad. Sci. U.S.A. 109, 15817-15822. doi: 10.1073/pnas.1205669109

Wang, B., and Li, Y. (2006). Evidence for the direct involvement of \{beta\} TrCP in Gli3 protein processing. Proc. Natl. Acad. Sci. U.S.A. 103, 33-38. doi: 10.1073/ pnas.0509927103
Wang, C., Pan, Y., and Wang, B. (2010). Suppressor of fused and Spop regulate the stability, processing and function of Gli2 and Gli3 full-length activators but not their repressors. Development 137, 2001-2009. doi: 10.1242/dev.052126

Yin, W. C., Satkunendran, T., Mo, R., Morrissy, S., Zhang, X., Huang, E. S., et al. (2019). Dual regulatory functions of SUFU and targetome of GLI2 in SHH subgroup medulloblastoma. Dev. Cell 48, 167.e5-183.e5. doi: 10.1016/j.devcel. 2018.11.015

Yung, T., Poon, F., Liang, M., Coquenlorge, S., McGaugh, E. C., Hui, C. C., et al. (2019). Sufu- and Spop-mediated downregulation of Hedgehog signaling promotes beta cell differentiation through organ-specific niche signals. Nat. Commun. 10:4647. doi: 10.1038/s41467-019-12624-5

Zhang, Q., Shi, Q., Chen, Y., Yue, T., Li, S., Wang, B., et al. (2009). Multiple Ser/Thr-rich degrons mediate the degradation of Ci/Gli by the Cul3-HIB/SPOP E3 ubiquitin ligase. Proc. Natl. Acad. Sci. U.S.A. 106, 21191-21196. doi: 10.1073/ pnas.0912008106

Zhang, Q., Zhang, L., Wang, B., Ou, C. Y., Chien, C. T., and Jiang, J. (2006). A hedgehog-induced BTB protein modulates hedgehog signaling by degrading Ci/Gli transcription factor. Dev. Cell 10, 719-729. doi: 10.1016/j.devcel.2006. 05.004

Zhang, Z., Shen, L., Law, K., Zhang, Z., Liu, X., Hua, H., et al. (2017). Suppressor of fused chaperones gli proteins to generate transcriptional responses to sonic hedgehog signaling. Mol. Cell Biol. 37, MCB.421-MCB.416. doi: 10.1128/MCB. 00421-16

Zhuang, M., Calabrese, M. F., Liu, J., Waddell, M. B., Nourse, A., Hammel, M., et al. (2009). Structures of SPOP-substrate complexes: insights into molecular architectures of BTB-Cul3 ubiquitin ligases. Mol. Cell 36, 39-50. doi: 10.1016/j. molcel.2009.09.022

Conflict of Interest: The authors declare that the research was conducted in the absence of any commercial or financial relationships that could be construed as a potential conflict of interest.

Publisher's Note: All claims expressed in this article are solely those of the authors and do not necessarily represent those of their affiliated organizations, or those of the publisher, the editors and the reviewers. Any product that may be evaluated in this article, or claim that may be made by its manufacturer, is not guaranteed or endorsed by the publisher.

Copyright (c) 2021 Umberger and Ogden. This is an open-access article distributed under the terms of the Creative Commons Attribution License (CC BY). The use, distribution or reproduction in other forums is permitted, provided the original author(s) and the copyright owner(s) are credited and that the original publication in this journal is cited, in accordance with accepted academic practice. No use, distribution or reproduction is permitted which does not comply with these terms. 